\title{
Philosophiques
}

\section{Armstrong, David M. Les Universaux. Une introduction partisane, trad. de l'anglais par Stéphane Dunand, Bruno Langlet et Jean-Maurice Monnoyer, Paris, Les éditions d'Ithaque, coll. " Science et Métaphysique ", 2010, 208 p.}

\section{Ghislain Guigon}

Volume 38, numéro 1, printemps 2011

URI : https://id.erudit.org/iderudit/1005732ar

DOI : https://doi.org/10.7202/1005732ar

Aller au sommaire du numéro

Éditeur(s)

Société de philosophie du Québec

ISSN

0316-2923 (imprimé)

1492-1391 (numérique)

Découvrir la revue

Citer ce compte rendu

Guigon, G. (2011). Compte rendu de [Armstrong, David M. Les Universaux. Une introduction partisane, trad. de l'anglais par Stéphane Dunand, Bruno Langlet et Jean-Maurice Monnoyer, Paris, Les éditions d'Ithaque, coll. " Science et Métaphysique ", 2010, 208 p.] Philosophiques, 38(1), 331-336.

https://doi.org/10.7202/1005732ar d'utilisation que vous pouvez consulter en ligne. 


\title{
Comptes rendus
}

\author{
Armstrong, David M. Les Universaux. Une introduction partisane, \\ trad. de l'anglais par Stéphane Dunand, Bruno Langlet et Jean- \\ Maurice Monnoyer, Paris, Les éditions d'Ithaque, coll. «Science \\ et Métaphysique», 2010, $208 \mathrm{p}$.
}

Ce livre de David M. Armstrong, destiné aux enseignants et aux étudiants, est sans doute la meilleure introduction aux débats contemporains sur le problème des universaux par un auteur qui a joué un rôle primordial dans le renouvellement de ces débats et le regain d'intérêt de la communauté philosophique pour ce thème classique. La traduction française de ce classique de la métaphysique analytique par Stéphane Dunand, Bruno Langlet et JeanMaurice Monnoyer respecte fidèlement le style clair et lucide de l'auteur.

La structure de l'ouvrage est la suivante. Le premier chapitre est une brève introduction au problème des universaux dans laquelle Armstrong critique les formes dites "extrêmes » du nominalisme: celles qui n'admettent pas de division objective entre des classes naturelles de particuliers et des classes de particuliers qui ne sont pas naturelles. Ce chapitre introductif est suivi de deux chapitres critiques portant chacun sur une version du nominalisme austère «modéré »: le nominalisme des classes naturelles (chapitre 2) et le nominalisme de la ressemblance (chapitre 3). Les deux chapitres suivants sont consacrés à des variétés du réalisme des universaux: le chapitre 4 discute et critique la conception des particuliers comme faisceaux d'universaux attribuée à Bertrand Russell; dans le chapitre 5, Armstrong expose, après avoir critiqué une conception platonicienne du réalisme des universaux, sa conception des universaux comme manières d'être des particuliers. Le chapitre 6 , qui précède un récapitulatif final, est consacré à la théorie des tropes, forme libérale du nominalisme, qu'Armstrong rejette.

Le livre d'Armstrong est suivi en annexe de deux articles récents de l'auteur qui fournissent une réelle valeur ajoutée à l'édition en français par rapport à l'édition originale en anglais. Le premier de ces articles, "Quatre disputes sur les propriétés", prend une perspective plus générale sur la métaphysique des propriétés. L'apport principal de cet article est selon moi la discussion portant sur le débat entre les conceptions catégoriques des propriétés et les conceptions des propriétés comme pouvoirs ou dispositions. Ce débat, qui est aujourd'hui l'un des plus vivants en métaphysique des propriétés, est en effet absent de l'introduction d'Armstrong et méritait d'être intégré à l'ouvrage. Le second article en annexe, "Les particuliers ont leurs propriétés par nécessité », montre judicieusement en quoi le réalisme d'Armstrong a évolué ces dernières années. Dans cet article, Armstrong pré- 
sente une conception provocante et stimulante de l'instanciation des universaux par les particuliers comme identité partielle entre un universel et un particulier - sur laquelle je reviendrai plus bas.

Des particuliers numériquement distincts semblent appartenir à un même type. «Le» et «le» sont deux occurrences du même type de mot (p. 13-14); différentes particules appartiennent au type des électrons; Bucéphale et Rossinante sont des chevaux, etc. Le problème des universaux nous est posé par le fait que des choses numériquement distinctes semblent avoir quelque chose en commun, semblent appartenir à un même type. Y répondre, c'est donc, selon Armstrong (chapitre 1), prendre une position philosophique à propos de cette apparente communauté de type par des choses distinctes: est-elle authentique et objective? Et comment en rendre compte? L'introduction d'Armstrong se concentre sur des solutions qui répondent positivement à la première de ces questions: la communauté de type par des particuliers distincts est authentique et objective (voir en particulier la page 27 du chapitre 1). On peut reprocher ce parti pris d'emblée réaliste, car il existe des solutions anti-réalistes au problème des universaux qui mériteraient d'être présentées dans une introduction au problème ${ }^{1}$. Les forces en présence admettant toutes que des particuliers distincts peuvent objectivement appartenir à un même type, la discussion se concentre sur l'analyse à donner à ce "même »: parlons-nous ici d'identité au sens strict?

Selon Armstrong les universaux ne font pas partie du problème, mais de la solution. Des électrons distincts semblent avoir quelque chose en commun parce qu'ils ont réellement quelque chose qui leur est commun, qui leur est identique: une ou plusieurs propriétés universelles. La position d'Armstrong est essentiellement présentée dans le chapitre 5 du livre et est développée dans l'article "Les particuliers ont leurs propriétés par nécessité», en annexe.

Armstrong conçoit les particuliers comme des entités "structurées" (chapitre 2, p. 53), c'est-à-dire comme possédant des propriétés. Cette conception est motivée par le rejet des formes austères de nominalisme que sont le nominalisme des classes (chapitre 1), le nominalisme des classes naturelles (chapitre 2) et le nominalisme de la ressemblance (chapitre 3) selon lesquelles les particuliers sont «amorphes ». Je reviendrai sur certaines raisons de l'opposition d'Armstrong à ces formes de nominalisme par la suite. Les particuliers étant conçus comme structurés, les propriétés qu'ils possèdent peuvent soit être des universaux qu'ils partagent au sens strict (chapitres 4 et 5) soit des propriétés particulières, dites "tropes", qui leur sont spécifiques mais sont exactement similaires (chapitre 6), la ressemblance exacte des tropes jouant le rôle de tofu nominaliste à l'identité des universaux à travers leurs instances.

1. Voir en particulier Barry Taylor «Natural Properties in Metaphysics ", Mind 102/405 (janvier 1993), p. 81-100. 
Les propriétés étant admises, nous pouvons ou bien concevoir les particuliers comme n'étant réellement rien de plus que des structures ou «faisceaux» de propriétés comprésentes, ou bien concevoir les propriétés comme des attributs d'un particulier qui leur est distinct. Si les propriétés sont conçues comme des universaux, alors la première option est, selon Armstrong, insatisfaisante (chapitre 5). Le rejet par Armstrong de la théorie des faisceaux associée à un réalisme des universaux est principalement motivé par son opposition au principe d'identité des indiscernables. Ce principe étant rejeté, il pourrait y avoir des particuliers numériquement distincts partageant toutes et seulement leurs propriétés. Or, si les particuliers ne sont que des faisceaux d'universaux, nous devrions conclure que les indiscernables sont identiques, car ils ne sont rien d'autre que des structures identiques d'universaux numériquement identiques.

Une théorie des faisceaux qui conçoit les propriétés comme des tropes ne souffre pas du même défaut puisque les indiscernables se révèlent être, selon cette théorie, des structures de propriétés numériquement distinctes bien qu'exactement similaires. Cependant, Armstrong préfère une théorie des tropes qui conçoit les tropes comme des attributs de particuliers à une théorie des particuliers comme faisceaux de tropes. L'argument avancé par Armstrong est que les tropes ne sont pas «taillés pour être les substances du monde» (chapitre 6, p. 137). En effet, les substances sont conçues comme des entités susceptibles d'avoir une existence indépendante (chapitre 4, p. 91-92), tandis que les tropes, dans un faisceau, ne peuvent exister indépendamment des autres tropes du faisceau: il n'y a pas de tropes flottants. Cet argument d'Armstrong est néanmoins peu convaincant. Armstrong a sûrement raison de penser que les tropes ne sont pas taillés pour jouer le rôle des substances ainsi définies, mais de cela il suit seulement qu'il n'y a pas de substance (au sens défini) selon la théorie des faisceaux: le monde est un monde d'entités dépendantes. Aussi vénérable que soit la catégorie des substances, rien n'indique en effet qu'une métaphysique viable ne puisse s'en dispenser.

Armstrong conçoit les particuliers comme structurés, avec des propriétés qui sont des attributs «immanents» des particuliers. Ainsi, il admet un principe d'instanciation des universaux selon lequel il ne peut y avoir d'universaux non instanciés: il n'y a pas de ciel platonicien où les universaux attendraient que des particuliers y accrochent leur grappin d'instanciation (chapitre 5, p. 95-102). Il n'y a pas non plus de particuliers "nus", de particuliers n'instanciant aucune propriété (chapitre 5, p. 114-115). Ainsi, le monde est un "monde d'états de choses", de particuliers instanciant des propriétés, ces dernières étant conçues comme universelles. La solution au problème des universaux défendue par Armstrong est donc que des particuliers sont d'un même type en vertu du fait qu'ils instancient un même - au sens strict d'identité — universel. 
D'après Armstrong, le problème principal auquel fait face sa théorie est l'explication des ressemblances entre propriétés. La théorie explique pourquoi des particuliers se ressemblent (parce qu'ils partagent un ou plusieurs universaux), mais différentes propriétés semblent elles-mêmes appartenir à des types communs et se ressembler. Pourquoi le vermillon est-il plus similaire au carmin qu'au bleu ciel? Pourquoi les différentes charges électriques appartiennent-elles à un même type de propriétés auquel n'appartiennent pas les propriétés que sont la masse et la couleur ? Ce problème conduit Armstrong à concevoir les universaux similaires comme des universaux structuraux, c'est-à-dire des universaux qui sont des structures complexes d'universaux, tels qu'ils partagent un ou plusieurs éléments de leur structure. Mais comme la littérature récente l'a montré, cette solution est intenable, et le problème de la ressemblance des universaux semble inextricable ${ }^{2}$.

Un autre problème de la théorie d'Armstrong telle qu'elle apparaît dans le livre concerne la relation d'instanciation dont l'aspect ésotérique semble difficile à éliminer. Le domaine des relations se divise en deux catégories: les relations internes qui sont telles que, certains termes étant dotés de certaines natures, ces relations obtiennent nécessairement entre ces termes et les relations externes dont l'obtention entre des termes n'est pas uniquement déterminée par la nature de ces termes (chapitre 3, p. 59-60). Mais l'instanciation n'est d'aucune de ces deux catégories. Le réaliste ne peut admettre qu'elle est externe sans tomber sous le coup de la «régression de Bradley »; mais elle n'est pas non plus interne, si l'instanciation d'un universel par un particulier est, dans le cas des propriétés accidentelles du moins, un lien contingent (chapitre 5, p. 129-132). L'ontologie d'Armstrong devient richement fournie à un niveau quasi inacceptable: elle contient des particuliers, des universaux, et ce lien magique d'instanciation qui n'entre dans aucune de ces catégories.

Une autre difficulté de la théorie d'Armstrong relative à l'instanciation a à voir avec l'une de ses objections contre le nominalisme de la ressemblance. La relation de ressemblance a certaines propriétés formelles: elle est réflexive, symétrique et non transitive. D'après Armstrong, le nominaliste de la ressemblance, puisqu'il prend celle-ci comme un primitif inanalysable, ne peut expliquer pourquoi la ressemblance a ces propriétés. Cela constitue, selon lui, un défaut important de cette forme de nominalisme puisque les théories rivales peuvent expliquer les propriétés de la ressemblance (chapitre 3, p. 55-593). Mais que dire de l'instanciation? L'instanciation d'un

2. Voir le livre de John Heil, D’un point de vue ontologique, traduction de l'anglais par Dominique Berlioz et François Loth, à paraître aussi aux Éditions d'Ithaque en avril 2011, sections 14.5 et 14.6; et l'excellent article de Maya Eddon, "Armstrong on Quantities and Resemblance ", Philosophical Studies 136/3 (2007), p. 385-404.

3. Pour une réponse, voir Gonzalo Rodriguez-Pereyra, Resemblance Nominalism: A Solution to the Problem of Universals (Oxford, Clarendon Press, 2002), p. 69-79. 
universel par un particulier est irréflexive, asymétrique et intransitive. Le réaliste des universaux peut-il expliquer pourquoi l'instanciation a ces propriétés? Il semble que la réponse doit être négative. Dans ce cas, l'objection d'Armstrong contre le nominalisme de la ressemblance frappe avec la même force la théorie qu'il défend. ${ }^{4}$

Armstrong tente de répondre à ces objections à propos de l'instanciation dans l'article «Les particuliers ont leurs propriétés par nécessité », judicieusement proposé en annexe. Dans cet article, il présente une nouvelle conception de l'instanciation d'un universel par un particulier. Selon cette conception, l'instanciation d'un universel par un particulier dans un état de chose, par exemple l'état de chose $a$ étant $F$, doit être conçue comme une identité partielle entre le particulier $a$ et l'universel $F$ (p. 187). Ainsi conçue, l'instanciation est telle que l'universel instancié est une partie du particulier (ibid.) qui l'instancie, tout comme le particulier est une partie de l'universel qu'il instancie. Le particulier $a$ et l'universel $F$ instancié par $a$ sont des entités qui, au sens strict, se chevauchent du fait qu'elles ont une partie commune: l'état de chose $a$ étant $F$. L'avantage de cette modification est que l'identité et le chevauchement méréologique n'ont rien de mystérieux: ce sont des relations formelles internes, totalement déterminées par la nature de leurs termes. Bien sûr, nous devons encore admettre comme axiomes les propriétés formelles de l'identité partielle: il s'agit d'une relation réflexive, symétrique et non transitive. Mais ceux-ci sont des axiomes de la logique et de la méréologie que toute métaphysique semble devoir admettre.

Reste un certain nombre de problèmes. Manifestement, l'identité partielle a des propriétés formelles fort différentes de celles accordées au départ à l'instanciation: d'une relation irréflexive, asymétrique et intransitive, l'instanciation devient, s'il s'agit vraiment d'identité partielle, une relation réflexive, symétrique et non transitive. Un tel changement ne peut être sans conséquence pour la théorie. L'une de ces conséquences est la suivante. Armstrong admet que, si l'instanciation d'un universel par un particulier est conçue comme de l'identité partielle, alors les particuliers ont leurs propriétés par nécessité. Cela est dû au fait que l'identité partielle est une relation interne et que le particulier et l'universel sont des sortes de touts méréologiques. Si vous enlevez une partie $x$ à un tout $y$, alors, selon la méréologie extensionnelle, $y$ cesse d'exister; l'existence d'un tout étant déterminé par celle de ses parties. Ainsi, si l'universel $F$ est une partie du particulier $a$, il n'est pas possible que $a$ existe sans instancier $F$ (p. 188). Cela semble être une couleuvre difficile à avaler si nous tenons à la distinction

4 Pour une autre réponse de ce type «Tu quoque!» aux objections d'Armstrong, voir David K. Lewis, "Une nouvelle tâche pour une théorie des universaux ", traduction de l'anglais par Ghislain Guigon, Concepts et catégories, Numéro spécial des Cahiers philosophiques de Strasbourg 17 (2004), p. 345-404, en particulier p. 359-365. 
entre propriétés contingentes et propriétés essentielles d'un particulier. Mais Armstrong a le gosier large et l'avale.

La couleuvre semble trop grosse lorsqu'on se souvient de l'un des arguments d'Armstrong contre le nominalisme des classes naturelles qu'il estime fatal contre cette théorie: l'argument de Wolterstorff (chapitre 2, p. 41-42). Selon le nominalisme des classes naturelles, deux particuliers appartiennent à un même type en vertu de leur appartenance à une même classe naturelle. Les conditions d'identité des classes sont telles que l'identité d'une classe est déterminée par ses membres: des classes sont distinctes si et seulement si elles ont des membres distincts. Rossinante et Rucio (le mulet de Sancho Panza) appartiennent au type des équidés. Supposez que Rossinante vienne à mourir dans l'un des combats épiques de Don Quichotte, alors Rossinante, n'existant plus, n'est plus un membre de la classe naturelle des équidés. Nous avons donc affaire à une classe différente des équidés, ce qui implique que le type des équidés est différent et donc que la nature de Rucio a changé. Cela semble inacceptable. Parallèlement, les conditions d'identité des touts ne sont pas très différentes de celles des classes: changez une partie du tout, et vous obtenez un tout différent. Supposez donc que Rucio soit au départ un mulet bien nourri de $400 \mathrm{~kg}$. Selon la conception de l'instanciation comme identité partielle, les universaux "être un mulet» et «avoir une masse de $400 \mathrm{~kg}$ » ont une partie commune: Rucio. Supposez maintenant que Rucio, amaigri par ses aventures, ne pèse plus que $300 \mathrm{~kg}$. Dans ce cas, le Rucio de $400 \mathrm{~kg}$ est un particulier numériquement distinct du Rucio de $300 \mathrm{~kg}$ puisque les particuliers ont leurs propriétés par nécessité. Mais alors, puisque l'universel «être un mulet » est lui-même partiellement identique aux particuliers qui l'instancient, un changement dans ses parties implique logiquement que l'universel «être un mulet» soit différent. Ainsi, affamer un mulet suffit à engendrer un nouveau type d'équidés, cela semble tout à fait inacceptable.

Bien qu'Armstrong prenne parti pour le réalisme des universaux dans cette introduction, l'ouvrage, essentiellement argumentatif, est plus militant que partisan. À la lecture du livre, le sentiment qui domine est qu'Armstrong nous propose un réalisme des universaux faute de mieux : c'est parce que les théories rivales sont insatisfaisantes qu'il faut se tourner vers un réalisme des universaux. Certains lecteurs seront convaincus par les arguments d'Armstrong, et alors l'ouvrage ouvre la voie à des recherches intéressantes sur la ressemblance des universaux et la nature de l'instanciation. D'autres seront renforcés dans leur incrédulité à l'égard du réalisme des universaux à la lecture de ce livre. Dans ce cas, les critiques d'Armstrong contre les nominalismes offrent un formidable programme de recherche. Dans tous les cas, ce livre est une référence essentielle pour tous ceux et celles que les débats abstraits de métaphysique ne rebutent pas.

GHISLAIN GUIGON 